\title{
Localization and Tracking Control Using Hybrid Acoustic-Optical Communication for Autonomous Underwater Vehicles
}

\author{
Ding Zhang ${ }^{12}$, Ibrahima N'Doye ${ }^{1}$, Member, IEEE, Tarig Ballal ${ }^{1}$, Member, IEEE, Tareq Y. \\ Al-Naffouri ${ }^{1}$, Senior, IEEE, Mohamed-Slim Alouini ${ }^{1}$, Fellow, IEEE, and \\ Taous-Meriem Laleg-Kirati ${ }^{1}$ Senior, IEEE
}

\begin{abstract}
This paper studies the problem of localization and tracking of a mobile target ship with an autonomous underwater vehicle (AUV). A hybrid acoustic-optical underwater communication solution is proposed, in which the acoustic link is used for the non-line-of-sight (NLoS) localization, and the optical link is for the line-of-sight (LoS) transmission. By coordinating these two complementary technologies, it is possible to overcome their respective weaknesses and achieve accurate localization, tracking, and high-rate underwater data transmission. The main challenge for reliable operation is to maintain the AUV over an optical link range while the target dynamics is unknown at all times. Hence, we design an error-based adaptive model predictive controller (MPC) and a proportional-derivative (PD) controller incorporating a real-time acoustic localization system to guide the AUV towards the sensor node mounted on the surface ship. We define a connectivity threshold cone with its apex coinciding with the sensor node such that when the underwater vehicle stays inside of this cone, a minimum bit rate is guaranteed. The localization, tracking control and optical communication scheme are validated through online simulations that integrate a realistic AUV model where the effectiveness of the proposed adaptive MPC and PD controller are demonstrated.
\end{abstract}

Index Terms-Localization, positioning, acoustic communication, optical communication, autonomous underwater vehicle, angle-of-arrival, phase-difference method, adaptive model predictive controller, proportional derivative controller.

\section{INTRODUCTION}

Recently, there has been a growing interest in exploring the underwater environment for various applications such as studying climate change, oceanic animals, monitoring oil rigs, surveillance, and autonomous operations [1]. These applications have given rise to a demand for reliable and efficient communication solutions in an underwater environment.

Today, multiple terrestrial communication technologies have been extensively studied for underwater applications. The most commonly utilized ones are based on radio frequency (RF) channels, acoustic channels, and optical channels.

Research was carried out in the past to study the feasibility of low-frequency RF and acoustic underwater wireless communications (UWC) [2]-[5]. These communication techniques

1 Computer, Electrical and Mathematical Sciences and Engineering Division (CEMSE), King Abdullah University of Science and Technology (KAUST), Thuwal 23955-6900, Saudi Arabia (email: ibrahima.ndoye @ kaust.edu.sa, tarig.ahmed@kaust.edu.sa, tareq.alnaffouri@kaust.edu.sa slim. alouini@kaust.edu.sa taousmeriem.laleg@kaust.edu.sa).

${ }^{2}$ Department of Electronic and Computer Engineering, The Hong Kong University of Science and Technology, Clear Water Bay, Kowloon, Hong Kong, China. (email: ding.zhang@ connect.ust.hk)

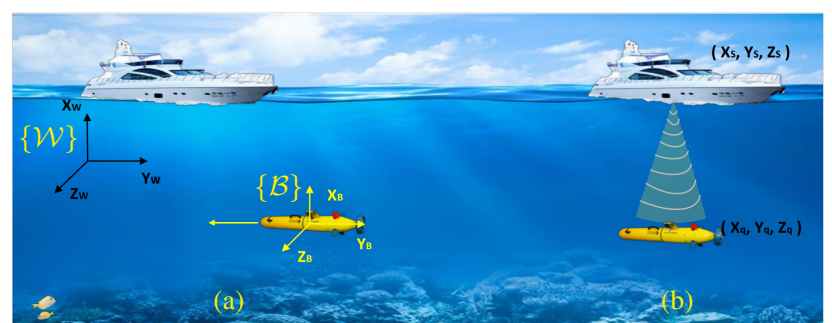

Fig. 1: (a) Acoustic communication is used by an AUV to move closer to the surface ship equipment sensor. (b) Optical communication link is used once within this range for highdata transfer.

are suitable for non-line of sight applications with a high tolerance to delay on account of their long transmission range. However, they are limited to relatively low data rates and high power consumption in timely data transmission tasks.

Underwater optical wireless communications (UOWC) enables high-speed underwater transmission within a limited communication range. Current research on UOWC focuses on increasing the data rate and transmission range of UOWC systems for different types of water [2], [6], [7]. Methods proposed in [1], [8]-[11] improves data rates of UOWC systems by using light-emitting diodes (LEDs). The proposed LED-based UOWC systems achieve Mbps-level data rates in pure seawater, though the transmission distance is restricted by the radiation angle and light intensity distribution.

Alternatively, hybrid systems comprising both acoustic and UWOC systems are introduced in [12]-[15] where systems can switch between optical and acoustic channels to adapt to varying communication ranges. Hybrid communication systems extend the applicability of underwater wireless communication to scenarios well beyond the restriction resulting from the tradeoff between data rate and transmission range. Subsequently, underwater monitoring missions can be costly due to the high cost of marine devices. Hence, the deployed communication system should be highly reliable to avoid communication failure and loss of the underwater vehicle [5], [14], [16], [17]. For marine tasks in a specific range, a solution with a higher data rate, robustness, and reliability is of significant interest.

The design of reliable tracking controllers is challenging due to the nonlinearities in AUV dynamics and the uncertainties in 
the ocean environment [18], [19]. Most existing works address a particular aspect of AUV trajectory tracking performance and focus on relatively simple mapping [18]-[26]. Typically, these methods do not consider the fact that the AUV may also need to collect and deliver large real-time volumes of data such as videos, images, or sensor readings. Besides, most of trajectory tracking results for AUV assume that a preplanned trajectory of the surface ship described in global coordinates is available to the AUV in real-time [18]-[26], through either GPS or broadcasting of the surface vessel. For underwater scenario, such an assumption is, unfortunately, not always valid in practical underwater applications.

In this paper, we consider a setup with a target ship and an AUV, both equipped with a pair of hybrid acoustic-optical transceivers. The AUV is blind to the dynamics of the target ship. Therefore, real-time localization is needed to form the feedback loop. The acoustic link carries out low bandwidth transmission serving for localization purposes, and the optical link is used for high rate transfer. Fig. 11 (a) illustrates an AUV moving towards a surface ship sensor node while estimating their relative position. Once the AUV reaches a cone-shaped area specified by the model of the optical communication channel, the optical channel is used to carry out high data rate transfer with guaranteed quality of service (QoS) (see Fig. 1.b)). The acoustic localization is executed in parallel to lock the AUV inside the cone with the optical communication, and its updated frequency is adjusted to a lower level to reduce the power consumption while maintaining desirable QoS.

To the best of the authors' knowledge, designing a hybrid acoustic-optical scheme for the localization and trajectory tracking control of a mobile target ship with an AUV based on the estimate position error in the context of collecting and carrying out rapid data exchange in real-time underwater wireless communication platform has not been thoroughly investigated, and one of the motivations of the paper is to bridge this gap. The novelty of our proposed solution is the fact that only the estimate of position error is used as the reference for the trajectory tracking controller of the AUV. In contrast, most of the existing tracking control approaches require the global reference trajectory. Furthermore, this work paves the way for constructing completed auxiliary trajectory tracking control of AUVs systems for maintaining stable underwater optical communication links and contributes to more extensive optical communication scenarios, such as underwater freespace optical communications, etc.

The main contributions of the paper are summarized as follows.

- A hybrid acoustic-optical scheme for the localization and trajectory tracking of a mobile target ship with an AUV based on the estimate of position error is designed.

- The proposed algorithm is introduced in the context of pairing a mobile target ship with an AUV to collect and carry out rapid data exchange in real-time underwater wireless communication platform.

- The novelty of our proposed solution lies in the fact that only the estimate of position error can be effectively used as the reference for the trajectory tracking control system of the AUV.
- Two different control strategies based on adaptive MPC and PD controller are tested through online simulations that integrate a real-time acoustic localization system and a realistic model of the hybrid acoustic-optical communication link.

- The performance results show that the adaptive MPC is featured by the input constraint handling capability and can deal with complex nonlinearity and parametervarying in the AUV systems dynamics. Moreover, the simulation results reveal the prominent robustness aspects of the adaptive MPC.

The remainder of this paper is organized as follows. Section III details the acoustic and the optical wireless link models of this hybrid communication system. Section III explains the phase-based methodology to localize the target. Section IV evaluates the performance of the two proposed controllers through numerical simulations using MATLAB/Simulink to achieve favorable tracking within competitive times. Finally, section $\mathrm{V}$ presents concluding remarks.

\section{COMmunicATion Channels}

In this paper, we present channel models for the acoustic and optical links separately. These models are implemented in MATLAB/Simulink to simulate the acoustic signal propagation and the variation of the data rate of the optical channel while the state of the system is evolving.

\section{A. Underwater Acoustic Channel}

We outline the propagation of the underwater acoustic channel model under additive phase noise and multiplicative signal strength attenuation. The surface ship is equipped with an omnidirectional anchor, while the AUV has omnidirectional anchor nodes symmetrically deployed at the end of each of its arms.

As the acoustic link is used for relatively low data rate tasks, we applied a sinusoidal acoustic signal $x_{\mathrm{s}}(t)$, at a single frequency $f_{\mathrm{Ac}}$, as an acoustic source emitted from the ship node, that is:

$$
x_{\mathrm{s}}(t)=A_{0} \sin \left(2 \pi f_{\mathrm{Ac}} t+\phi_{0}\right),
$$

where $A_{0}$ and $\phi_{0}$ are the source amplitude and initial phase, respectively.

As shown in Fig. 2, a sensor array composed of four acoustic sensors is mounted on the AUV sampling and measuring the acoustic signal. The four sensors are arranged in two orthogonal pairs; each pair is used to estimate the AoA of the signal relative to the sensor pair baseline. The relative position between the ship and AUV is the constraint in a particular range. At the same time, the operational speed of the surface vessel is relatively slow compared to the propagation speed of sound in water. Hence the Doppler effect of sound is not considered in this setup, and the received signal is given as follows

$$
x_{\mathrm{r} i}(t)=A \sin \left(2 \pi f_{\mathrm{Ac}} t+\phi_{i}+\phi_{w i}+\phi_{0}\right)+w_{i}(t),
$$

where $i=1, \cdots, 4$ indices the four sensor nodes of the AUV. $A$ is the amplitude of the received signal, $\phi_{i}$ is a phase shift 


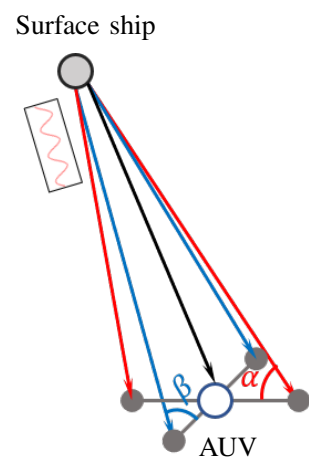

Fig. 2: Illustration of the two angle-of-arrival estimation principle.

due to the propagation delay, $\phi_{w i}$ is a phase uncertainty, and $w_{i}(t)$ is an additive zero-mean Gaussian random noise.

The attenuation of acoustic signal strength is frequency dependent and can be written as follows [27]

$$
10 \log _{10}\left(\frac{A_{0}}{A}\right)^{2}=\kappa \cdot 10 \log _{10} d+d \cdot 10 \log _{10} \bar{\alpha},
$$

where $\kappa$ represents the geometric spreading factor which is usually bounded between 1 and $2, d$ is the distance between the acoustic transmitter and receiver and $\bar{\alpha}$ is the absorption coefficient. To compute the value of $\bar{\alpha}$, we use the Thorp's formula as follows [28], [29]

$10 \cdot \log _{10} \bar{\alpha}=\frac{0.1 f^{2}}{1+f^{2}}+\frac{40 f^{2}}{4100+f^{2}}+2.75 \times 10^{-4} \cdot f^{2}+0.003$,

where $f=f_{\mathrm{Ac}}$ is the carrier frequency.

As mentioned earlier, the phase shift is the result of the propagation delay. The propagation speed of the underwater acoustic channel model is not constant but depends on the salinity, temperature, depth, etc. [30], [31]. Consequently, the propagation path is not a straight line but a curve. However, these factors only have a small impact on sound speed over a short-range. Thus, we assume that sound speed is approximately constant and is taken as $v_{a}=1500 \mathrm{~m} / \mathrm{s}$.

\section{B. Underwater Optical Link}

Most efficient optical wireless communication systems adopt intensity modulation with direct detection (IM/DD), which provides high-speed ranges for a variety of applications through on-off Keying (OOK) in the physical layer [32]. We assume a channel model of a directed LoS optical IM/DD system [32]. The parameters of this optical link model are illustrated in Fig. 3

The radiant intensity of the optical transmitter is given by [32], 33]

$$
I_{s}(d, \phi)=P_{\mathrm{TX}} \frac{m+1}{2 \pi d^{2}} \cos ^{m} \phi,
$$

where $\phi$ represents the angle between the heading of the transmitter and the optical link, $d$ is the length of the optical link, $m$ denotes the Lambert's mode number counting the effect of the source beam, and $P_{\mathrm{TX}}$ describes the average

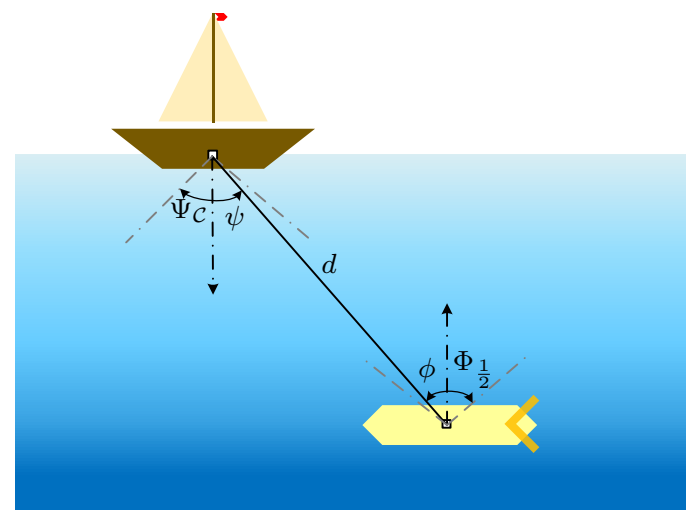

Fig. 3: Main parameters of the optical link model.

transmitted optical power. The mode number is computed using [32], [33]

$$
m=\frac{-\ln 2}{\ln \left(\cos \Phi_{1 / 2}\right)},
$$

where $\Phi_{1 / 2}$ is the half-angle at half-power of average transmitted optical source that describes the transmitter beam width.

Let $A_{\text {eff }}$ denote the effective area of the optical receiver collecting the incident photo radiation. It is related to the incident angle $\psi$. This area is given as follows

$$
A_{\text {eff }}(\psi)=f(\psi) A_{r} \cos \psi, \quad|\psi| \leqslant \Psi_{\mathcal{C}}
$$

where $A_{r}$ is the receiver active area, and $f(\psi)$ is the light concentrator gain given by

$$
f(\psi)= \begin{cases}\frac{n^{2}}{\sin ^{2} \Psi_{\mathcal{C}}} & \text { if }|\psi| \leqslant \Psi_{\mathcal{C}} \\ 0 & \text { if }|\psi|>\Psi_{\mathcal{C}} .\end{cases}
$$

Here, $\Psi_{\mathcal{C}}$ is the half-angle field-of-view (FOV) of the optical detector and $n$ is the refractive index of the sea water.

Light propagation in seawater is associated with high attenuation due to absorption and scattering that depend on the properties of the light beam and water. We use an approximately exponential attenuation model, which provides a sufficiently accurate estimate of the optical power in clear ocean waters where absorption is predominant [34], [35]. Since our aim here is to estimate the position of the AUV within optical communication channel, especially when the surface ship equipment sensor is unknown and the distance is considerable; we settle the formulation of the channel loss as follows

$$
L_{\mathrm{ch}}=\exp \left(-K_{a} d\right)
$$

where $K_{a}$ is the diffuse attenuation coefficient [34].

Using (5), (7) and (9), the optical signal strength in $\mathrm{dB}$ at the detector side can be calculated as [32]

$$
P_{\mathrm{OW}}=10 \log _{10}\left(I_{s} A_{\text {eff }} L_{\mathrm{ch}}\right)+\xi,
$$

where $\xi$ is the mean of noise (in $\mathrm{dB}$ ) produced by natural light. This radiation power is described as being independent of the received signal, spectrally constant, and Gaussian [32], [36]. As illustrated in Fig. 3, we assume that $\phi=\psi$, i.e. the optical beam transceiver mounted on the AUV is always pointing up, while the one on the surface ship keeps pointing down. 
TABLE I: Optical Link Parameters.

\begin{tabular}{|c||cccc|}
\hline Transmitter & $P=0.25 \mathrm{~W}$ & $K_{a}=0.15 \mathrm{~m}^{-1}$ & $\Phi_{1 / 2}=20^{\circ}$ & $\Delta \lambda=30 \mathrm{~nm}$ \\
\hline Receiver & $A_{r}=1 \mathrm{~cm}^{2}$ & $\Psi_{\mathcal{C}}=30^{\circ}$ & $n=1.52$ & $R=0.7 \mathrm{~A} / \mathrm{W}$ \\
\hline
\end{tabular}

\section{Connectivity Cone}

Figs. $4 \mathbf{a}$ ) and $4 \mathbf{b}$ ) show the contour maps of the bit rate of the optical link as a function of the relative position of the target ship and the AUV when the BER level is set to $10^{-4}$. Table II summarizes the parameters used for creating these plots.

Now, combining this range with the maximum receiver pointing error that yields $\psi=\Psi_{\mathcal{C}}$, a half-sided circular cone called connectivity cone, as shown in Fig. $5 \mathrm{a}$ ) can be completely defined. Hence, a received signal strength greater than or equal to the desired one would be sufficiently guaranteed if the transmitter lies in this cone.

The apex of $\mathcal{C}$ is given by the position of the ship on the water surface. Since the ship transceiver points down at all times, the normal vector of the cone is always perpendicular to the horizontal plane and pointing into the sea. Furthermore, $\mathcal{C}$ can be specified entirely by its half aperture angle of $\Psi_{\mathcal{C}}$ and by its slant height $d_{\mathcal{C}}$. Since $I_{\mathrm{b}}$ is a transcendental function of $d$, the value of the slant height $d_{\mathcal{C}}$ of $\mathcal{C}$ is evaluated numerically through the distribution of $B$ along the link length $d$ as shown in Fig. 5b). Finally, we can find that this bit rate $B=10 \mathrm{Mbps}$ which is equivalent to " 7 " in logarithm scale is achievable at a range of $d_{\mathcal{C}} \approx 7.9680 \mathrm{~m}$. Hence, we obtain that the height $h$ of the cone which is $h_{\mathcal{C}}=d_{\mathcal{C}} \times \cos \Psi_{\mathcal{C}}=6.9005 \mathrm{~m}$.

\section{Methodology}

In this section, we firstly describe the dynamical model of the AUV. Then, we propose an adaptive model predictive control (MPC) controller based on successive online linearization of the nonlinear AUV model and a PD controller to follow a desired direction towards the surface ship sensor node. In the end, we present the proposed method for estimating the direction vector error based on the phase-differences measured by the four acoustic sensor nodes.

\section{A. Autonomous Underwater Vehicle (AUV) Model}

We define a body-fixed frame $\{\mathcal{B}\}$ and an earth-fixed frame $\{\mathcal{W}\}$ for the AUV motion description, as shown in Fig. 6. The dynamics of the AUV in $\{\mathcal{B}\}$ reference is given as [37], [38]

$$
M \dot{\nu}+C(\nu) \nu+D(\nu) \nu+g(\eta)=\tau+\tau_{w}
$$

where $\eta$ and $\nu$ are the position and velocity of the AUV in $\{\mathcal{W}\}$ and $\{\mathcal{B}\}$ references, respectively. $M$ is the inertia matrix, $C(\nu)$ represents the matrix of Coriolis' and centripetal terms and $D(\nu)$ is the damping matrix. $\tau$ is the vector of the input signals, $g(\eta)$ is an unknown vector of restoring forces and $\tau_{w}$ represents the vector of external input (force/torque) disturbances. Table II provides the main parameters of the AUV model [38].
TABLE II: Nomenclature.

\begin{tabular}{|c||c||c|}
\hline Symbol & Description & Unit \\
\hline$x$ & Position in surge & $\mathrm{m}$ \\
$\varrho$ & Position in sway & $\mathrm{m}$ \\
$\varrho$ & Yaw & $\mathrm{rad}$ \\
\hline$u$ & Linear velocity in surge & $\mathrm{ms}^{-1}$ \\
$v$ & Linear velocity in sway & $\mathrm{ms}^{-1}$ \\
$r$ & Yaw velocity & $\mathrm{rads}^{-1}$ \\
\hline$\tau_{1}$ & Force in surge & $\mathrm{N}$ \\
$\tau_{2}$ & Moments in yaw & $\mathrm{Nm}$ \\
$\tau_{w_{1}}$ & Disturbance in surge & $\mathrm{N}$ \\
$\tau_{w_{2}}$ & Disturbance in sway & $\mathrm{N}$ \\
$\tau_{w_{3}}$ & Disturbance in yaw & $\mathrm{Nm}$ \\
\hline$m$ & Weight of the AUV & $\mathrm{kg}^{2}$ \\
$I_{z}$ & Moments of inertia in yaw & $\mathrm{kgm}^{2}$ \\
$L$ & Length of AUV & $\mathrm{m}$ \\
\hline$X_{\dot{u}}$ & Added mass in surge & $\mathrm{kg}$ \\
$Y_{\dot{v}}$ & Added mass in sway & $\mathrm{kg}^{2}$ \\
$N_{\dot{r}}$ & Added mass in yaw & $\mathrm{kgm}^{2}$ \\
\hline$X_{u}$ & Linear damping coefficients in surge & $\mathrm{kgs}^{-1}$ \\
$Y_{v}$ & Linear damping coefficients in sway & $\mathrm{kgs}^{-1}$ \\
$N_{r}$ & Linear damping coefficients in yaw & $\mathrm{kgm}^{2} \mathrm{~s}^{-1}$ \\
\hline$X_{u|u|}$ & Quadratic damping coefficients in surge & $\mathrm{kgm}^{-1}$ \\
$Y_{v|v|}$ & Quadratic damping coefficients in sway & $\mathrm{kgm}^{-1}$ \\
$N_{r|r|}$ & Quadratic damping coefficients in yaw & $\mathrm{kgm}^{2}$ \\
\hline
\end{tabular}

The AUV formation is operating in the horizontal plane at a constant depth such as ocean floor applications. Then,

$$
\begin{aligned}
& \eta=\left[\begin{array}{lll}
x & y & \varrho
\end{array}\right]^{T}, \nu=\left[\begin{array}{lll}
u & v & r
\end{array}\right]^{T}, \tau=\left[\begin{array}{lll}
\tau_{1} & \tau_{2} & \tau_{3}
\end{array}\right]^{T}, \\
& w=\left[\begin{array}{lll}
w_{1} & w_{2} & w_{3}
\end{array}\right]^{T}, g(\eta)=0, M=\left[\begin{array}{ccc}
m_{11} & 0 & 0 \\
0 & m_{22} & 0 \\
0 & 0 & m_{33}
\end{array}\right], \\
& D(\nu)=\left[\begin{array}{ccc}
d_{11} & 0 & 0 \\
0 & d_{22} & 0 \\
0 & 0 & d_{33}
\end{array}\right], C(\nu)=\left[\begin{array}{ccc}
0 & 0 & -m_{22} v \\
0 & 0 & m_{11} u \\
m_{22} v & -m_{11} u & 0
\end{array}\right],
\end{aligned}
$$

where $m_{11}=m-X_{\dot{u}}, m_{22}=m-Y_{\dot{v}}, m_{33}=I_{z}-N_{\dot{r}}$, $d_{11}=-X_{u}-X_{u|u|}|u|, d_{22}=-Y_{v}-Y_{v|v|}|v|$, and $d_{33}=$ $-N_{r}-N_{r|r|}|r|$ [38|-[40|.

The position of the AUV in initial coordinate frame $\mathcal{W}$ without any control input in sway direction can be described as

$$
\dot{\eta}=R(\varrho) \nu,
$$

where the transformation matrix $R$ is given as

$$
R(\varrho)=\left[\begin{array}{ccc}
\cos \varrho & -\sin \varrho & 0 \\
\sin \varrho & \cos \varrho & 0 \\
0 & 0 & 1
\end{array}\right] .
$$

The formulation of the AUV dynamics is transformed into $\mathcal{W}$ reference by using the kinematic transformations of the state variables and the model parameters as follows [41]

$$
\begin{aligned}
& \ddot{\eta}=R(\varrho) \dot{\nu}+\dot{R}(\varrho) \nu, \\
& M(\eta)=R^{-T}(\varrho) M R^{-1}(\varrho), \\
& C(\nu, \eta)=R^{-T}(\varrho)\left[C(\nu)-M R^{-1}(\varrho) \dot{R}(\varrho)\right] R^{-1}(\varrho),
\end{aligned}
$$



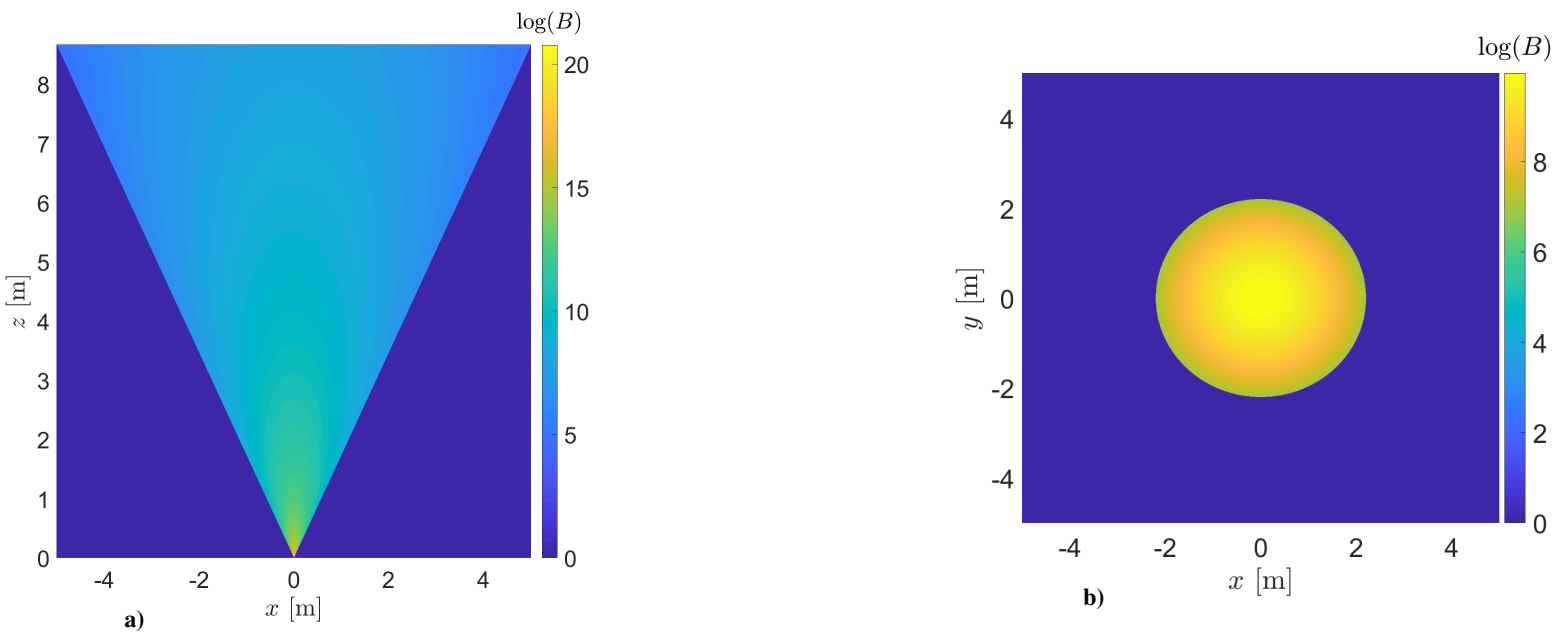

Fig. 4: Contour maps of bit rates: a) Side view; b) Top view.
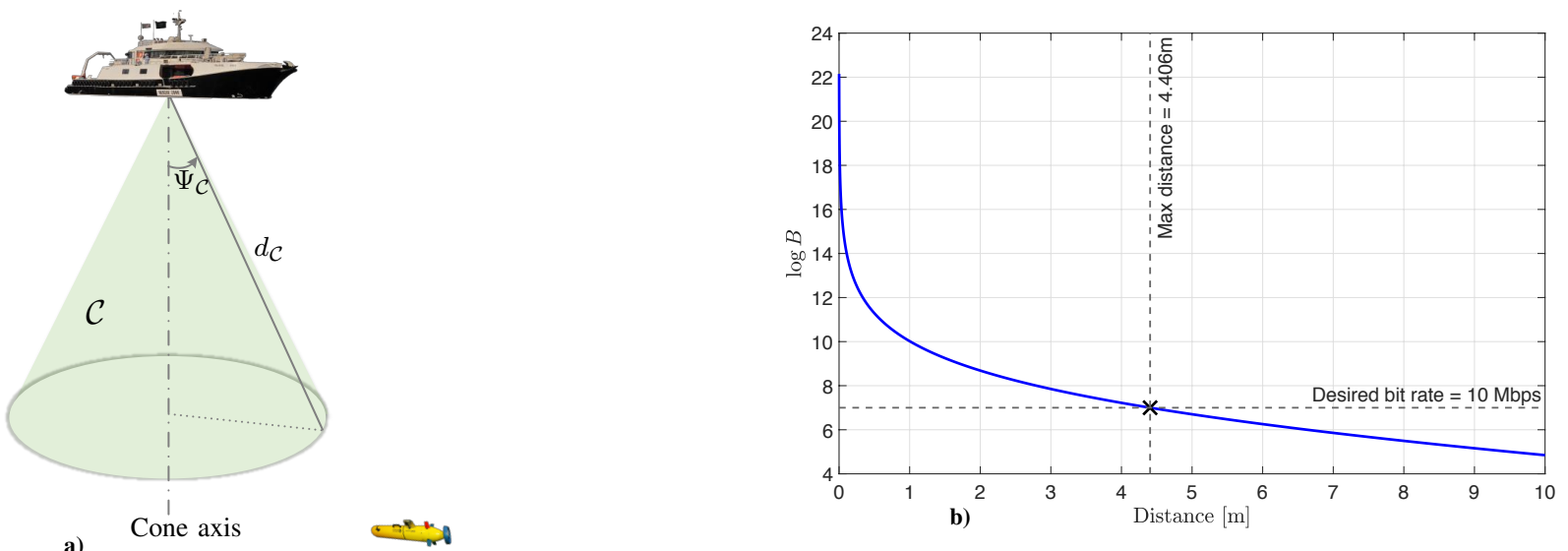

Fig. 5: a) Main parameters of the connectivity region $\mathcal{C}$; b) Value of the slant height $d_{\mathcal{C}}$ of $\mathcal{C}$.

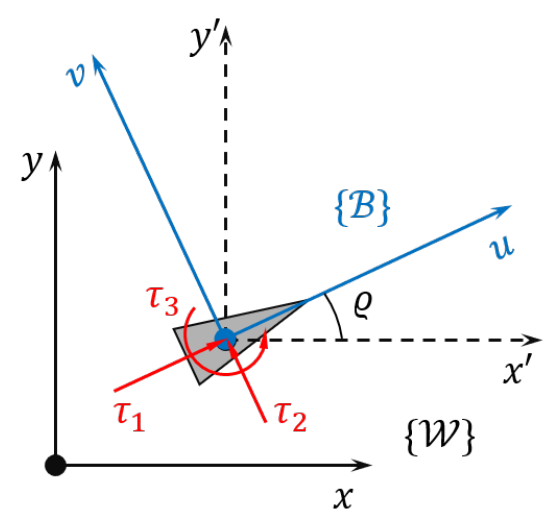

Fig. 6: Underwater vehicle model in horizontal plane.

$$
\begin{aligned}
& D(\nu, \eta)=R^{-T}(\varrho) D(\nu) R^{-1}(\varrho) \\
& \tau(\eta)=R^{-T}(\varrho) \tau .
\end{aligned}
$$

\section{B. Control Strategy}

Proportional derivative (PD) controller is the most used technique to control the position and orientation of dynamically positioned marine vehicles due to its design simplicity and good performance. However, it lacks better performance results in the presence of nonlinearity or disturbances in the system dynamics [42]. To overcome this drawback, we propose a linear parameter-varying (LPV) model predictive control (MPC) controller called adaptive MPC [43]-[45]. MPC computes a trajectory of controller output by solving an optimization problem as output horizon moves forward [46]. The proposed adaptive MPC is based on successive online linearization of the nonlinear AUV model. Here, the nonlinear AUV model 11 is linearized around the current operating point at each control horizon and a linear MPC controller is designed for the resulting system. MPC scheme uses nominal values of the parameters, while an adaptive MPC scheme allows to adjust the model online to compensate for timevarying process characteristics [47]. Our approach uses the acoustic transceiver signal to localize the relative position in $x-y$ plane for the AUV to the ship. Then, the estimated position, which is equivalent to the direction error vector, is fed to the closed-loop PD controller and the adaptive MPC. The resulting output then forces the AUV to enter the predefined connectivity cone and remain inside of the cone, i.e., establish and maintain the optical link with satisfactory QoS.

Let $\left[\begin{array}{lll}x_{\mathrm{RX}} & y_{\mathrm{RX}} & \varrho_{\mathrm{RX}}\end{array}\right]^{T}$ be the state of the ship transceiver, where $\eta_{\mathrm{RX}}=\left[\begin{array}{ll}x_{\mathrm{RX}} & y_{\mathrm{RX}}\end{array}\right]^{T}$ is the Cartesian coordinate of the surface ship transceiver in $x-y$ plane and $\varrho_{\mathrm{RX}}$ is its heading angle. We assume that the surface ship vehicle can change its 
heading angle $\varrho_{\mathrm{RX}}$, and moves around on the sea surface area.

The position of the AUV is given by $\eta_{\mathrm{Tx}}=$ $\left[\begin{array}{lll}x_{\mathrm{TX}} & y_{\mathrm{TX}} & \varrho_{\mathrm{TX}}\end{array}\right]^{T} \in \mathbb{R}^{3}$. Here, the external disturbances $\tau_{\omega}(\eta)$ are not considered in both controllers design. by

We propose a PD controller as a control tracking input given

$$
\tau=K_{p} \widetilde{\eta}+K_{v} \widetilde{\nu}
$$

where $K_{p}$ and $K_{v}$ are the control gains, $\widetilde{\eta}=\eta_{\mathrm{RX}}-\eta_{\mathrm{TX}}$ and $\widetilde{\nu}=\nu_{\mathrm{RX}}-\nu_{\mathrm{TX}}$ are the position and velocity errors, respectively.

Using equations (11), (12) and (13), the system dynamics can be formulated in $\{\mathcal{B}\}$ as follows

$M(\eta) \ddot{\eta}+[C(\nu, \eta)+D(\nu, \eta)] \dot{\eta}+g(\eta)=R^{-1}\left(\tau(\eta)+\tau_{\omega}(\eta)\right)$.

Let $\zeta:=[\widetilde{\eta} ; \dot{\vec{\eta}}] \in \mathbb{R}^{6}$ be the augmented error of the position and velocity errors. Considering that the cruising speed of a surface ship is typically around 20 knots $(\approx 15.4 \mathrm{~m} / \mathrm{s})$, then, the travel distance of the surface ship is $0.77 \mathrm{~m}$ for each control horizon given the control horizon $T_{c}=0.05 \mathrm{~s}$. As discussed in section II-C, the optical communication quality is guaranteed while the AUV enters the connectivity cone whose apex coincides with the optical transceiver mounted on the surface ship. In a particular depth, the interior of the cone is reduced the interior of a circle centered at the surface ship's projection onto the corresponding $x-y$ plane. Assume that the AUV is operating in the depth of $h_{\mathcal{C}}=6.9005 \mathrm{~m}$, the reduced connectivity circle has a radius of $h_{\mathcal{C}} \times \tan \Psi_{\mathcal{C}} \approx 3.984 \mathrm{~m}$ $>0.75 \mathrm{~m}$. Hence, we assume that the reference $\eta_{\mathrm{RX}}$ keeps constant during each control horizon, i.e., $\dot{\eta}_{\mathrm{Rx}}=0$. Therefore, we have

$$
\zeta=\left[\begin{array}{c}
\tilde{\eta} \\
\dot{\tilde{\eta}}
\end{array}\right]=\left[\begin{array}{c}
\eta_{\mathrm{Rx}}-\eta \\
-\dot{\eta}
\end{array}\right],
$$

then, the error dynamic can be described as follows

$$
\begin{aligned}
\dot{\zeta}= & \underbrace{\left[\begin{array}{cc}
0 & I \\
0 & M(\eta)^{-1}[C(\nu, \eta)+D(\nu, \eta)]
\end{array}\right]}_{A(\nu)} \zeta \\
& +\underbrace{\left[\begin{array}{c}
0 \\
-[R M(\eta)]^{-1}
\end{array}\right]}_{B(\nu)} \underbrace{\left(\tau(\eta)+\tau_{\omega}(\eta)\right)}_{u} .
\end{aligned}
$$

The control objective is to minimizes $\zeta$ from the estimated position error. Then, the adaptive MPC problem for the AUV trajectory tracking can be formulated as a quadratic optimization problem that is solved at each time $k_{c}=k N_{c},(k=$ $0,1, \cdots)$ to determine the control actions

$$
\begin{array}{r}
\min _{\substack{u_{k_{c}+i} \\
i=0, \cdots, N_{p}-1}} J_{k_{c}}(.)=\sum_{i=0}^{N_{p}-1}\left(\zeta_{k_{c}+i}^{T} Q \zeta_{t+i}+u_{k_{c}+i}^{T} P u_{k_{c}+i}\right) \\
+\zeta_{k_{c}+N_{p}}^{T} Q \zeta_{k_{c}+N_{p}}
\end{array}
$$

$$
\begin{array}{ll}
\text { s.t. } & \zeta_{k_{c}+i+1}=\zeta_{k_{c}+i}+\left(A\left(\nu_{k_{c}}\right) \zeta_{k_{c}+i}+B\left(\nu_{k_{c}}\right) u_{k_{c}+i}\right) T_{s}, \\
& u_{k_{c}+i+1}=u_{k_{c}+i}+\Delta u_{k_{c}+i}, \\
& \Delta u_{\min } \leqslant \Delta u_{k_{c}} \leqslant \Delta u_{\max },
\end{array}
$$

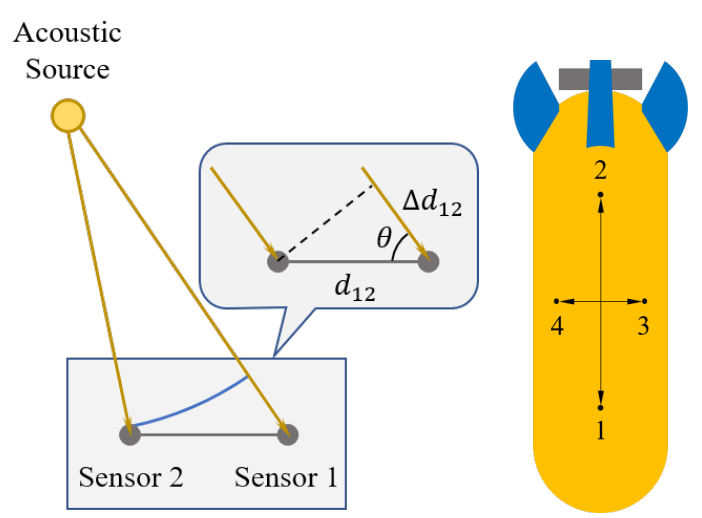

Fig. 7: Path difference of acoustic propagation and far field scenario.

$$
u_{\min } \leqslant u_{k_{c}} \leqslant u_{\max }
$$

where $\zeta$ is the tracking error vector, $u$ is the control input, $N_{p}$ is the prediction horizon and $N_{c}$ is the control horizon with $N_{p} \geqslant N_{c}$. The tuning matrices $P$ and $Q$ are semi-positive definite. The time discretization is derived using Euler approach and the constant sampling time $T_{s}$. The first $N_{c}$ elements of the resulting sequence $\left\{u_{k_{c}+i}\left(i=0, \cdots, N_{p}-1\right)\right\}$ is chosen as the output of the controller at the current control horizon. Here, the external disturbances $\tau_{\omega}(\eta)$ are not considered in the controller design.

\section{Direction Vector Estimation using Phase-Difference}

Wireless sensor networks (WSN) localization has been extensively used for acoustic tracking of underwater sound sources [48]-[52] and AoA is one of the major measurement techniques. The AoA estimation process can be implemented by measuring the phase difference of acoustic signals perceived by two or more sensors, which can be directly translated into direction vectors [27], [53], [54].

As described in section II-C, the acoustic propagation speed is assumed to be a constant $v_{a}=1500 \mathrm{~m} / \mathrm{s}$. In the setup, the largest distance between a sensor is $l=2 \mathrm{~m}$. Thus the critical distance to enter the far field of transmitter is around $\frac{l^{2}}{v_{a} / f_{\mathrm{Ac}}}=\frac{4}{1500 / 100} \approx 26.67 \mathrm{~cm}$; in our setup, the minimum distance between the ship and AUV is $\sim 3.8 \mathrm{~m}$ which is clearly a far field case as depicted in Fig. 7.

In the far field of an acoustic source, the incident paths of acoustic signal at a sensor pair are treated as parallel to each other. With such assumption, we then have the following approximation

$$
\Delta d_{12}=v_{a} \frac{\Delta \phi}{2 \pi f_{\mathrm{Ac}}} \approx d_{12} \cos \theta,
$$

where $\Delta \phi$ is given by the difference between Fast Fourier Transform (FFT)-based estimates of phases of signals captured by two coupled sensors. The equation then can be rewritten as follows

$$
\theta=\arccos \frac{v_{a} \Delta \phi}{2 \pi d_{12} f_{\mathrm{Ac}}} .
$$

The aforementioned orthogonal sensor setup and the phasedifference based AoA estimation output the value of two angles $\alpha$ and $\beta$ as depicted in Fig. 8 . 


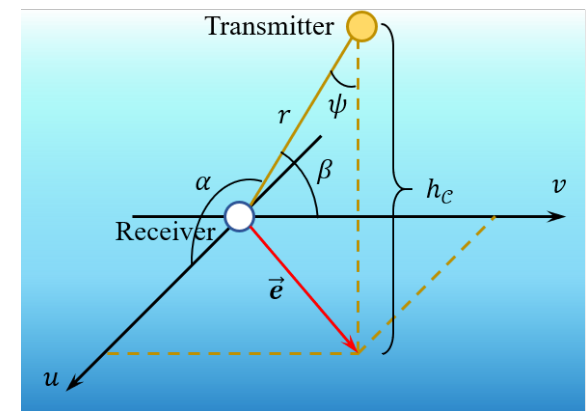

Fig. 8: Geometry of the localization method.

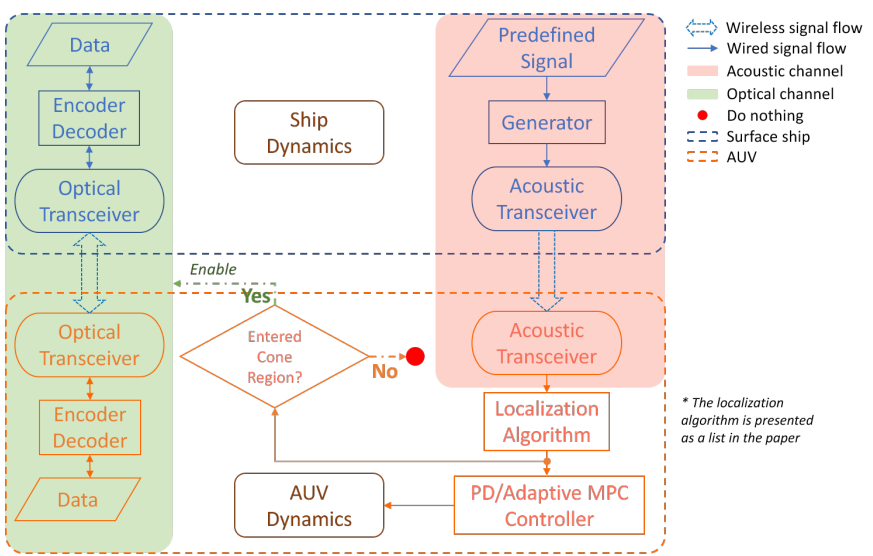

Fig. 9: Overall workflow of the proposed hybrid acousticoptical communication system.

Under the assumption that the AUV is operating at the same depth $h_{\mathcal{C}}$ and the position variables are in $\{\mathcal{B}\}$ frame, we calculate the relative position $\overrightarrow{\mathbf{e}}$ of AUV with respect to the surface ship using their geometric relationship.

$$
\begin{aligned}
& r=h_{\mathcal{C}} / \cos \psi, \quad|\cos \psi|=\sqrt{\sin ^{2} \alpha-\cos ^{2} \beta}, \quad \text { and } \\
& \overrightarrow{\mathbf{e}}=\left[\begin{array}{ll}
r \cos \alpha & r \cos \beta
\end{array}\right]^{T},
\end{aligned}
$$

where $r$ is distance between surface ship and the AUV.

By using 20, we derive the following relationship

$$
\overrightarrow{\mathbf{e}}=\left[\begin{array}{ll}
\frac{h_{\mathcal{C}} \cos \alpha}{\sqrt{\sin ^{2} \alpha-\cos ^{2} \beta}} & \frac{h_{\mathcal{C}} \cos \beta}{\sqrt{\sin ^{2} \alpha-\cos ^{2} \beta}}
\end{array}\right]^{T} .
$$

Fig. 9 demonstrates the overall scheme used in this paper. In summary, we present an acoustic localization method that is robust in water in a specific range. The obtained localization information is then transferred to the adaptive MPC and PD controller, which is used to align the AUV and the surface ship. Once their distance is estimated to be within a cone threshold, the high-speed optical communication channel is enabled. In such a way, the data rate and reliability of the whole system are well balanced.

\section{Simulation Results AND Discussions}

We apply all of our system simulations in MATLAB/Simulink environment and interpret the simulation results, which highlight the advantages of the proposed adaptive MPC and PD controller within the hybrid communication system. The Simulink model contains the AUV system dynamics,

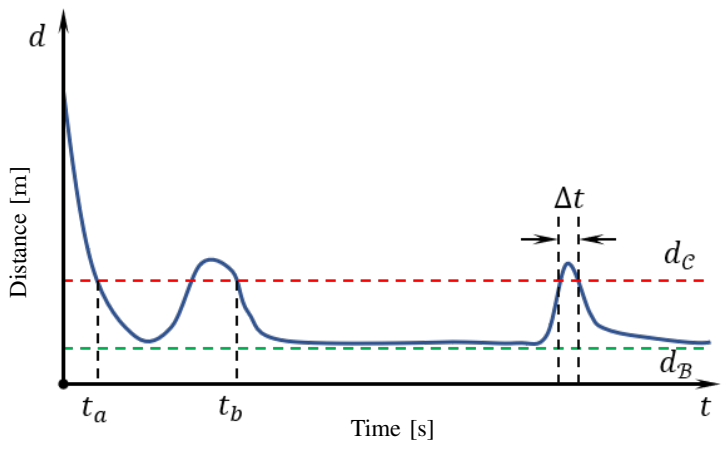

Fig. 10: Example of closed-loop stable time response of the system: $d_{\mathcal{C}}$ is the slant height of the cone, $d$ is the length of optical link, $d_{\mathcal{B}}$ is the minimum possible distance for an AUV working at a certain depth $h, d_{\mathcal{B}}=h$.

the adaptive MPC and PD controller, as well as the propagation model of the acoustic channel. The sampling time and the end time of the simulation are set to $0.005 \mathrm{~s}$ and $100 \mathrm{~s}$, respectively. The relative position, i.e., the position error of the AUV to the surface ship is calculated by the localization algorithm taking advantage of the acoustic channel at each time step. Then, the relative error is fed to the adaptive MPC and PD controller step by step as the simulation goes on. As we are dealing with a communication link performance, we also follow the performance metrics illustrated in Fig. 10 .

Cone Arrival Time $t_{a}$ : The time when the AUV enters the cone for the first time and the distance is less than the slant height of the connectivity cone $d_{\mathcal{C}}$ is defined as cone arrival time.

Communication Established Time $t_{b}$ : Ignoring the effect of external disturbances, the time when AUV enters the cone and stays inside after is defined as the communication established time.

Communication Restoring Time $\Delta t$ : Suppose an impulse input disturbance $\tau_{w}$ which might lead the AUV to out-of-cone status is introduced into the system. The time spent from the moment that the AUV gets out of the cone to the moment when AUV returns to the cone and stays inside after is defined as restoring time.

Root Mean Square Error $\mathrm{RMSE}_{\chi}$ : The $\mathrm{RMSE}_{\chi}$ values quantify the effect of the closed-loop steady state error performance after communication for a given interval of time to the two proposed controllers and permit us to conclude on the performance of the control strategy.

\section{A. Phase-based Localization Algorithm}

The algorithm is implemented and co-tested online with the adaptive MPC and PD controller in Simulink to mimic the sampling-based real-time application scenario. The sampling rate of the data acquisition device is set to $10 \mathrm{kHz}$. The acquisition device captures the four channels corresponding to each sensor in a pseudo-synchronous manner. Then, it transmits the stored data in its local buffer to the PC RAM buffer once the local buffer is filled to parse the time-varying phase information of each AUV sensor with lower channel delay. In PC RAM buffer, the block size is set to 500, i.e., 


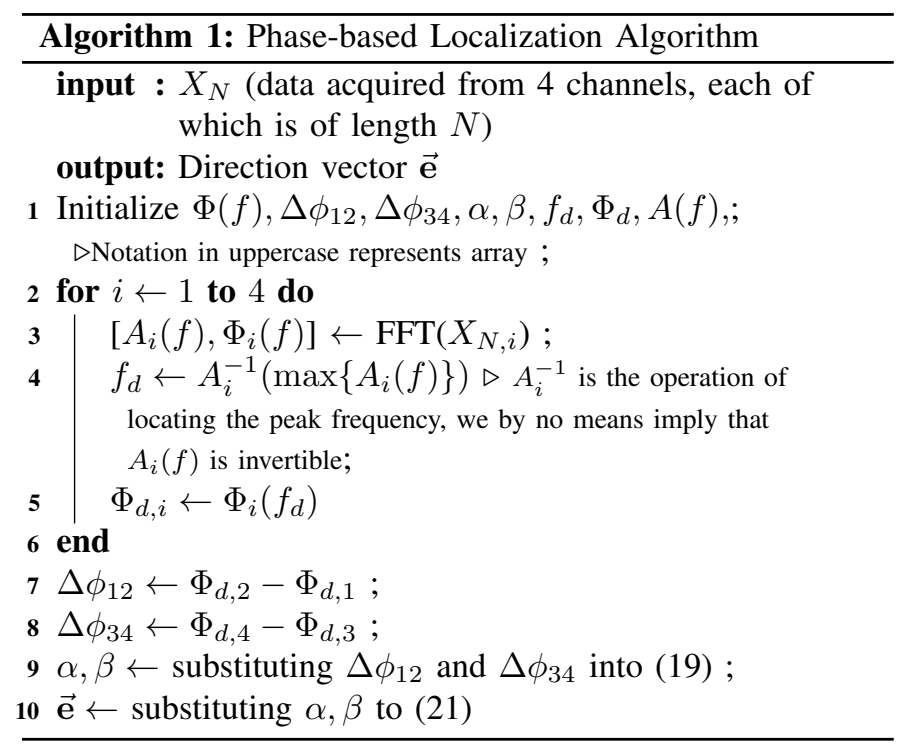

every 500 sampling points will generate an interrupt. The interrupt will then trigger the execution of algorithm 1, in which we use FFT to obtain the magnitude and phase spectrum of the captured discrete signal. The peak of the magnitude spectrum locates the dominant frequency, after which the corresponding phase information is extracted, and the phase difference between coupled sensors is calculated. Then, the direction vector for the time interval composed of these 500 sampling points is computed abiding by the formulas above. In such a case, the output of direction vector estimation is refreshed every 500 sampling points, which will slow the sampling rate down to $200 \mathrm{~Hz}$, neglecting the channel delay, transmission, and processing delay.

\section{B. Parameter Selection}

To test the performance of the two proposed target tracking controllers for underwater based optical communication through numerical simulations, we set the initial position of the transmitter at $\left[\begin{array}{lll}5 & -5 & 0\end{array}\right]^{T}$. At the same time, the mobile ship receiver is at the origin with a heading angle $\varrho_{\mathrm{RX}}=0^{\circ}$. The parameters of the system dynamics of the AUV are adapted from [38] and are given in Table [III. The transmitter-receiver distance is approximately $8.03 \mathrm{~m}$ at the beginning, and the control gains $K_{p}=\operatorname{diag}\{60,100,50\}$ and $K_{v}=\operatorname{diag}\{30,20,1\}$ are tuned empirically to obtain a fast, smooth response without overshoot. Let the ship be at $\left[\begin{array}{lll}0 & 0 & 0\end{array}\right]$ when the AUV starts to track the ship, and we make that the surface ship receiver follows a desired nonlinear curve to test the AUV tracking control. The nonlinear Bézier curve is well-used in the literature when it comes to realtime motion planning. This curve provides a lot of desired properties for curves in motion planning and is a popular approach for on-road driving scenarios.

\section{Target Tracking Performance in Nominal Conditions}

To force the AUV transmitter to reach the connectivity cone and then stay within it without assuming any external disturbances, we use the adaptive MPC and PD controller (14) to control the AUV. We make that the surface ship vehicle follows a Bezier curve trajectory. Then, the trajectories of the states with respect to time and $x-y$ plane described by the receiver and the transmitter using the adaptive MPC and PD controller without any external disturbances are shown in Figs. 11a) and 11b), respectively. The blue, red and green dashed curves are the simulated AUV trajectory using the adaptive MPC, the PD controller and the desired surface ship trajectory, respectively. As we can see, both adaptive MPC and PD controller drive the vehicle to the desired trajectory, which verifies the closed-loop tracking stability.

To test the performance of the two proposed controllers, we plot the distance $d$ that separates transmitter-receiver line, the logarithm of the bit rate $B$, and the receiver pointing error $\psi$ as shown in Figs. 12a), 12b), and 13, respectively. We note that after around $t_{a}=4 \mathrm{~s}$, the adaptive MPC outperforms the PD controller in terms of time responses. In addition, the distance of both adaptive MPC and PD controller is less than $d_{\mathcal{C}}$ and stays less this distance all along the simulation. Hence, the AUV remains within the connectivity cone from this time on and the bit rate is guarantee to be around $10 \mathrm{Mbps}$.

\section{Robust Test}

The inherent robustness to the external disturbances and measurement noises is an important factor for marine control systems. Therefore, we investigate the robustness of the tracking control in underwater based optical communication. To show the robustness of the adaptive MPC and PD controller for maintaining a perfect position between transmitter and receiver to establish a directed optical LoS link, we simulate the AUV tracking control under a strict test condition: ocean current forces of magnitude $\left[\begin{array}{llll}350 & (\mathrm{~N}) & 350(\mathrm{~N}) & 350(\mathrm{~N} . m)\end{array}\right]^{T}$ and measurement noises are introduced as disturbances. The ocean current disturbances occur at $30 \mathrm{~s}$ and last for $1 \mathrm{~s}$.

From the simulation results illustrated in Figs. 14a) and 14 b) with disturbances, we find that the adaptive MPC based tracking control leads the AUV well converged to the desired trajectory, while the tracking control with PD controller exhibits large tracking errors. The RMSE for the nominal and disturbance cases of the adaptive MPC and PD controller is summarized in Table IV] Roughly, the adaptive MPC demonstrates the tracking control performance, and the AUV converges faster to the desired trajectory.

To test the performance of the two proposed adaptive MPC and PD controller with model parameter error, measurement noises, and ocean currents forces, we plot the distance $d$ that separates the transmitter-receiver line, the logarithm of the bit rate $B$ and the receiver pointing error $\psi$ as shown in Figs. $15 \mathrm{a}), 15 \mathrm{~b})$, and 16 , respectively.

We note that around $t_{a}=30 \mathrm{~s}$ and $t_{a}=34 \mathrm{~s}$ the distance of the adaptive MPC and PD controller is less than $d_{\mathcal{C}}$ and stays less than this distance all along with the simulation, respectively. Hence, the AUV stays within the cone-shaped region from this time on, and the bit rate is guaranteed to be around $10 \mathrm{Mbps}$, as shown in Fig. 15b). Subsequently, when a short external input disturbance is applied to the system during 
TABLE III: Modeling Parameters of the AUV.

\begin{tabular}{|c|c|c|}
\hline$m_{11}=120 \mathrm{~kg}$ & $m_{22}=300 \mathrm{~kg}$ & $m_{33}=96 \mathrm{~kg}$ \\
\hline$d_{11}=(70+100|u|) \mathrm{kg} / \mathrm{s}$ & $d_{22}=(100+200|v|) \mathrm{kg} / \mathrm{s}$ & $d_{33}=(50+100|r|) \mathrm{kg} \cdot \mathrm{m}^{2} / \mathrm{s}$ \\
\hline
\end{tabular}
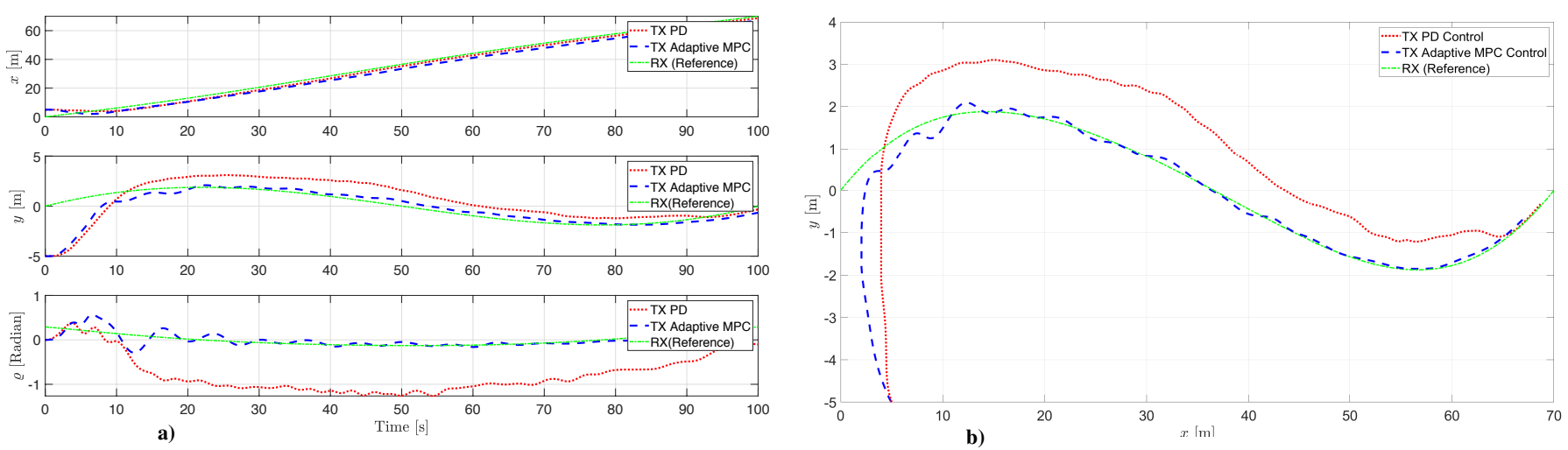

Fig. 11: a) States responses described by the AUV transmitter and the surface target ship receiver using adaptive MPC and PD controller without disturbances; b) 2D-view.
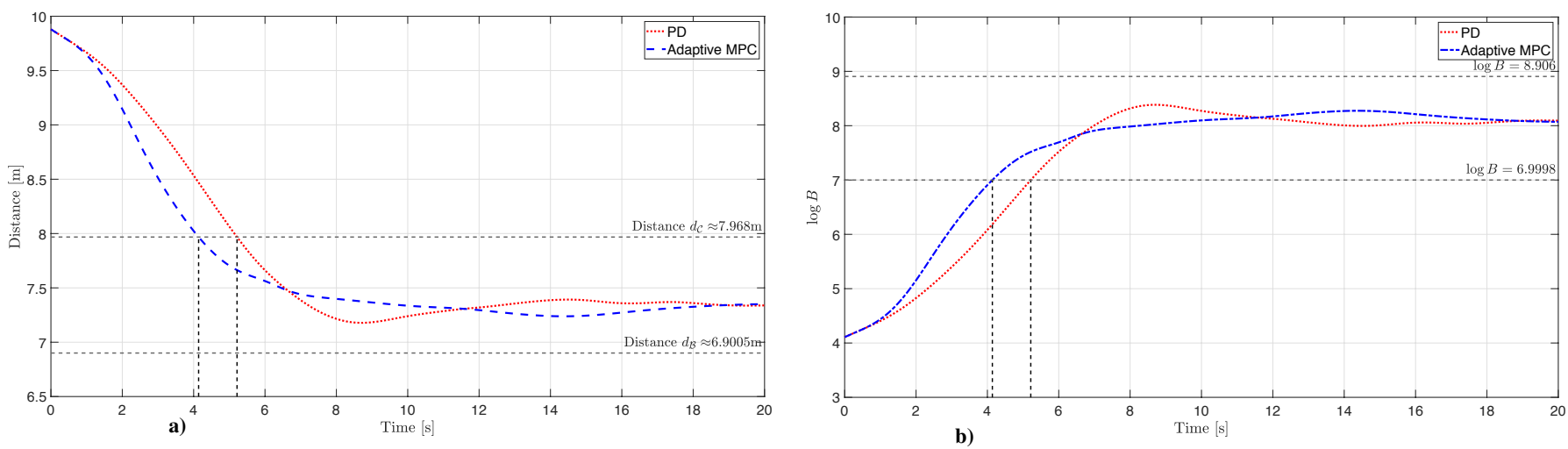

Fig. 12: a) Length of optical link $d$ (i.e., transmitter-receiver distance) using adaptive MPC and PD controller; b) Logarithm of the bit rate.

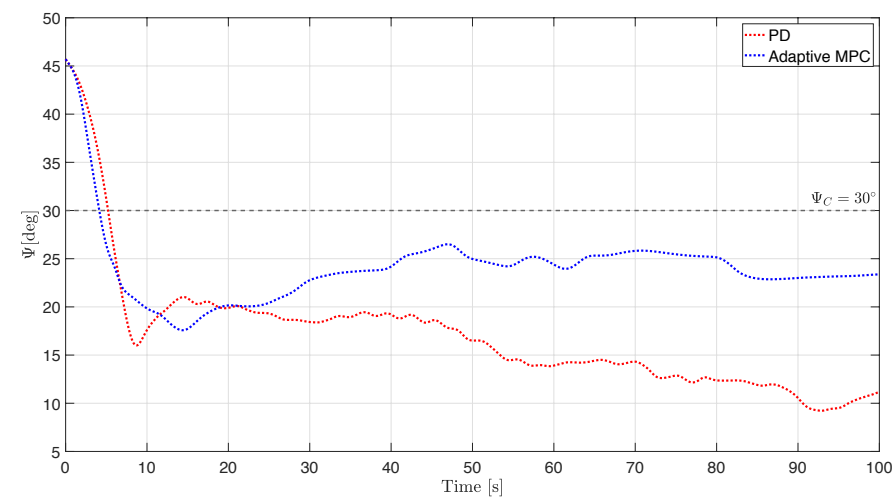

Fig. 13: Receiver pointing error $\psi$ : adaptive MPC versus PD controller

TABLE IV: RMSE Performance Comparison in Nominal and Disturbance Cases: Adaptive MPC versus PD controller.

\begin{tabular}{ccccc}
\hline Control scheme & $\mathrm{RMSE}_{x}[\mathrm{~m}] \mathrm{RMSE}_{y}[\mathrm{~m}] \mathrm{RMSE}_{\varrho}[\mathrm{rad}]$ & $t_{a}[\mathrm{~s}]$ \\
\hline PD controller & 1.628 & 1.201 & 0.860 & 5.216 \\
Adaptive MPC controller & 2.932 & 0.658 & 0.107 & 4.139 \\
PD with disturbances & 2.436 & 1.344 & 0.889 & 5.215 \\
Adaptive MPC (disturbances) & 2.890 & 0.682 & 0.356 & 4.139 \\
\hline
\end{tabular}

the communication link, the adaptive MPC has a much smaller $\Delta t$ and overshoot while PD controller lacks to maintain the communication link.

Finally, our objective of establishing and keeping an optical communication link and a positioning tracking performance between the surface ship receiver and the AUV transmitter with an average bit rate of $10 \mathrm{Mbps}$ is well accomplished using the adaptive MPC and PD controller. 

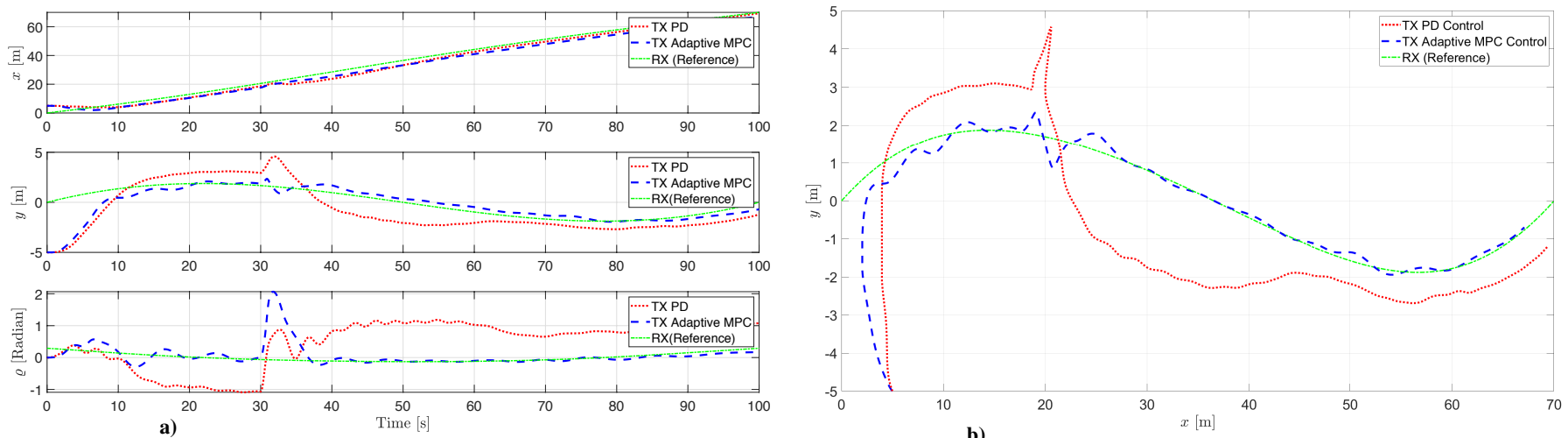

Fig. 14: a) States responses described by the AUV transmitter and the surface ship receiver using adaptive MPC and PD controllers with disturbances; b) 2D-view.
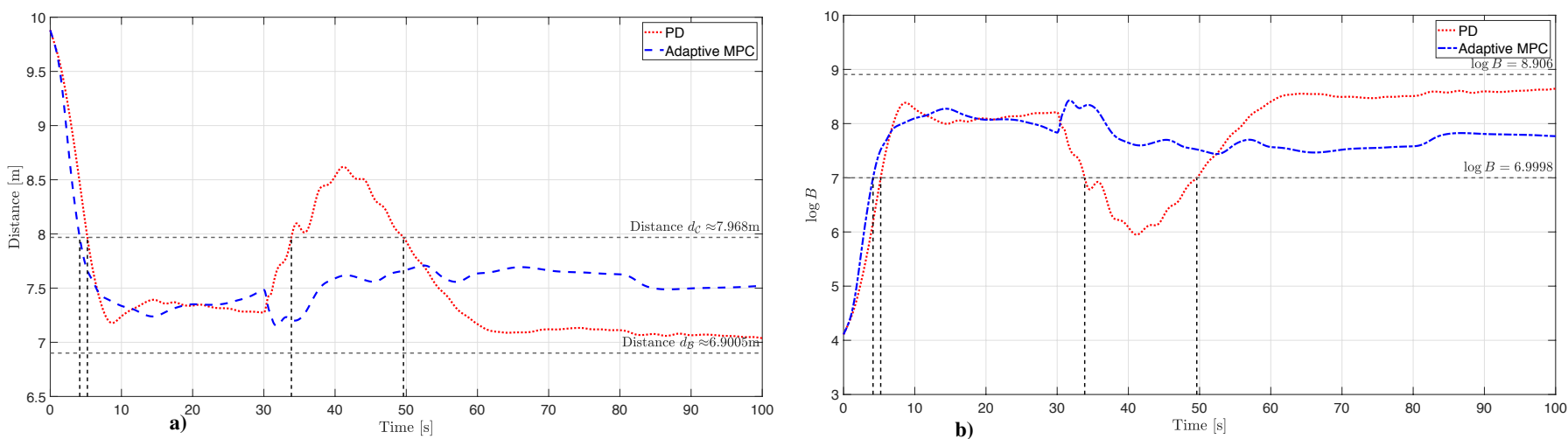

Fig. 15: Adaptive MPC and PD controller with measurement noises and ocean current forces: a) Length of optical link $d$; b) Logarithm of the bit rate.

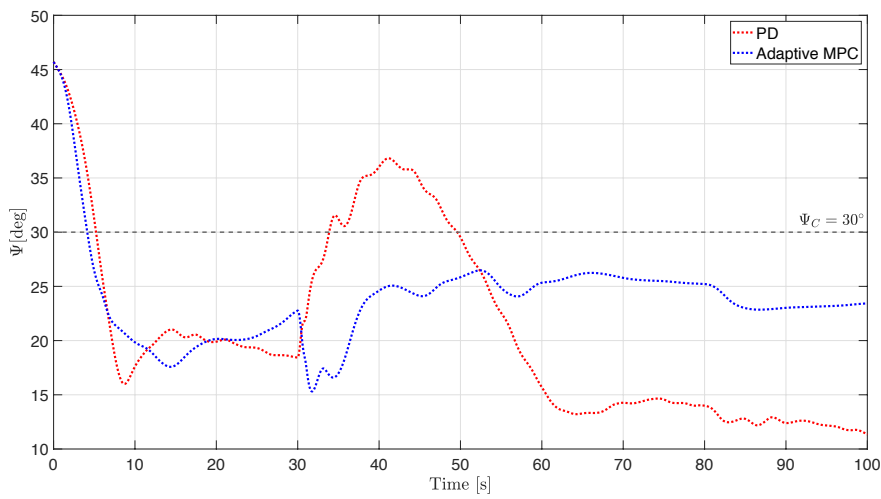

Fig. 16: Receiver pointing error $\psi$ : adaptive MPC versus PD controller.

\section{CONCLUSion}

In this work, a hybrid acoustic-optical scheme is designed for the AUV-based underwater wireless communication platform to carry out rapid data exchange in the water. We presented and demonstrated a comprehensive localization and derived two control strategies based on adaptive MPC and PD controller for the AUV system through numerical simulations. The key idea is to estimate position error using the phasedifferences measured by four acoustic directional sensors installed on the AUV at an acceptable update frequency, and then use it as the reference for the control system of the AUV. Once the optical signal strength is sufficient to reach the desired bit rate as well as BER, the AUV then heads for the optimal distance in its working depth, i.e., the vertical projection of the geometrical center of four ship sensor nodes onto the AUVs' working plane, while the optical channel is established and maintained.

In future work, we aim to validate experimentally our approach and instrument the lab-developed underwater communication platforms in a real underwater environment for testing. Meanwhile, we are planning to maximize the potential of our designed scheme by extending it to the network of a group of AUVs.

\section{ACKNOWLEDGMENT}

Research reported in this publication has been supported by research funding from King Abdullah University of Science and Technology (KAUST). 


\section{REFERENCES}

[1] J. S. Jaffe, "Underwater optical imaging: The past, the present, and the prospects," IEEE J. Ocean. Eng., vol. 40, no. 3, pp. 683-700, 2015.

[2] N. Saeed, A. Celik, T. Y. Al-Naffouri, and M. S. Alouini, "Underwater optical wireless communications, networking, and localization: A survey," Ad Hoc Networks, vol. 94, p. 101935, 2019.

[3] M. Stojanovic, "Recent advances in high-speed underwater acoustic communications," IEEE Journal of Oceanic Engineering, vol. 21, no. 2, pp. $125-136,1996$.

[4] M. Chitre, S. Shahabudeen, and M. Stojanovic, "Underwater acoustic communications and networking: Recent advances and future challenges," Marine Technology Society Journal, vol. 42, no. 1, pp. 103-116, 2008.

[5] F. Akyildiz, D. Pompili, and T. Melodia, "Underwater acoustic sensor networks: Research challenges," Ad Hoc Networks, vol. 3, no. 3, pp. 257-279, 2005.

[6] J. R. D. Retamal, H. M. Oubei, B. Janjua, Y. Chi, H. Wang, C. Tsai, T. K. Ng, D. Hsieh, H. Kuo, M. Alouini, J. He, G. Lin, and B. S. Ooi, "4-Gbit/s visible light communication link based on 16-QAM OFDM transmission over remote phosphor-film converted white light by using blue laser diode," Optics Express, vol. 23, no. 26, pp. 33656-33666, 2015.

[7] K. Otradnov, S. Volodymyr, and S. Kornieiev, "Underwater wireless video communication in operations of AUV/UUVs - new horizon of underwater explorations," Underwater Technologies, vol. 06, pp. 46-56, 2017.

[8] F. Hanson and S. Radic, "High bandwidth underwater optical communication," Appl. Opt., vol. 47, no. 2, pp. 277-283, 2008.

[9] S. Arnon, "Underwater optical wireless communication network," Opt. Eng., vol. 49, no. 6, pp. 1-15, 2010.

[10] M. Doniec, M. Angermann, and D. Rus, "An end-to-end signal strength model for underwater optical communications," IEEE J. Ocean. Eng., vol. 38, no. 4, pp. 743-757, 2013.

[11] C. Wang, H.-Y. Yu, Y.-J. Zhu, T. Wang, and Y.-W. Ji, "Multi-LED parallel transmission for long distance underwater VLC system with one SPAD receiver," Opt. Commun., vol. 410, no. 3, pp. 889-895, 2017.

[12] C. Lodovisi, P. Loreti, L. Bracciale, and S. Betti, "Performance analysis of hybrid optical/acoustic AUV swarms for marine monitoring," Fut. Internet, vol. 10, no. 7, pp. 1-18, 2018.

[13] C. Moriconi, G. Cupertino, S. Betti, and M. Tabacchiera, "Hybrid acoustic/optic communications in underwater swarms," in OCEANS, pp. 1-9, 2015.

[14] N. Saeed, A. Celik, T. Al-Naffouri, and M.-S. Alouini, "Energy harvesting hybrid acoustic-optical underwater wireless sensor networks localization," Sensors, vol. 18, no. 1, pp. 1-16, 2017.

[15] S. Han, Y. Noh, R. Liang, R. Chen, Y.-J. Cheng, and M. Gerla, "Evaluation of underwater optical-acoustic hybrid network," China Commun., vol. 11, no. 5, pp. 49-59, 2014.

[16] Z. Zeng, S. Fu, H. Zhang, Y. Dong, and J. Cheng, "A survey of underwater optical wireless communications," IEEE Communications Surveys and Tutorials, vol. 19, pp. 204-238, 2017.

[17] N. Farr, A. Bowen, J. Ware, C. Pontbriand, and M. Tivey, "An integrated, underwater optical /acoustic communications system," in IEEE Oceans'10, (Sydney, Australia), 2010.

[18] H. Li and W. Yan, "Model predictive stabilization of constrained underactuated autonomous underwater vehicles with guaranteed feasibility and stability," IEEE/ASME Transactions on Mechatronics, vol. 22, pp. 11851194, 2017.

[19] C. Shen and Y. Shi, "Distributed implementation of nonlinear model predictive control for auv trajectory tracking," Automatica, vol. 115, p. $108863,2020$.

[20] R. Cui, X. Zhang, and D. Cui, "Adaptive sliding mode attitude control for autonomous underwater vehicles with input nonlinearities," Ocean Engineering, vol. 123, pp. 45-54, 2016.

[21] T. Elmokadem, M. Zribi, and K. Youcef-Toumi, "Trajectory tracking sliding mode control of underactuated AUVs," Nonlinear Dynamics, vol. 84, no. 2, pp. 1079-1091, 2016.

[22] C. Shen, Y. Shi, and B. Buckham, "Integrated path planning and tracking control of an AUV: A unified receding horizon optimization approach," IEEE/ASME Transactions on Mechatronics, vol. 22, pp. 1163-1173, 2017.

[23] C. Shen, Y. Shi, and B. Buckham, "Modified C/GMRES algorithm for fast nonlinear model predictive tracking control of AUVs," IEEE Transactions on Control Systems Technology, vol. 25, pp. 1896-1904, 2017.
[24] C. Shen, Y. Shi, and B. Buckham, "Lyapunov-based model predictive control for dynamic positioning of autonomous underwater vehicles," in IEEE International Conference on Unmanned Systems (ICUS), (Denver, Colorado, USA), 2017.

[25] C. Shen, Y. Shi, and B. Buckham, "Trajectory tracking control of an autonomous underwater vehicle using lyapunov-based model predictive control," IEEE Transactions on Industrial Electronics, vol. 65, pp. 5796$5805,2018$.

[26] J. Gurerrero, J. Torres, V. Creuze, and A. Chemori, "Adaptive disturbance observer for trajectory tracking control of underwater vehicles," Ocean Engineering, vol. 200, p. 107080, 2020.

[27] T. Ballal, Angle-of-arrival Based Ultrasonic 3-D Location for Ubiquitous Computing. PhD thesis, University College Dublin, Ireland, 2010.

[28] M. J. Bocus, D. Agrafiotis, and A. Doufexi, "Real-time video transmission using massive MIMO in an underwater acoustic channel," in IEEE Wireless Communications and Networking Conference, (Barcelona, Spain), 2018.

[29] M. C. Domingo, "Overview of channel models for underwater wireless communication networks," Physical Communication, vol. 1, no. 3, pp. $163-182,2008$

[30] A. Sehgal, I. Tumar, and J. Schónwálder, "Aquatools: An underwater acoustic networking simulation toolkit," in OCEANS, (Sydney), pp. 14, 2010.

[31] K. V. MacKenzie, "Nine-term equation for sound speed in the oceans," Acoustical Society of America Journal, vol. 70, pp. 807-812, 1981

[32] Z. Ghassemlooy, W. Popoola, and S. Rajbhandari, Optical Wireless Communications: System and Channel Modelling with MATLAB. Berlin: CRC Press, 1st ed., 2012.

[33] T. C. Shen, R. J. Drost, C. C. Davis, and B. M. Sadler, "Design of duallink (wide-and narrow-beam) LED communication systems," Optics Express, vol. 22, no. 9, pp. 11107-11118, 2014.

[34] C. Mobley, Light and Water: Radiative Transfer in Natural Waters. New York, NY, USA: Academic,, 1994.

[35] C. Gabriel, M. A. Khalighi, S. Bourennane, P. Léon, and V. Rigaud, "Monte-carlo-based channel characterization for underwater optical communication systems," IEEE/OSA J. Opt. Commun. Netw., vol. 5, no. 1, pp. 1-12, 2013.

[36] P. Cruz, B. M. Sadler, and R. Fierro, "Sensor localization using hybrid RF/optical wireless communications for an aerial data mule," in Proc. IEEE American Contr. Conf., (Boston, MA, USA), 2016.

[37] T. Fossen, Guidance and Control of Ocean Vehicles. New York, NY, USA: WileyInterscience, 1994.

[38] R. Cui, S. S. Ge, B. V. Ee-How, and Y. S. Choo, "Leader-follower formation control of underactuated autonomous underwater vehicles," Ocean Engineering, vol. 37, pp. 1491-1502, 2010.

[39] P. Aguiar and A. M. Pascoal, "Dynamic positioning and way-point tracking of underactuated AUVs in the presence of ocean currents," International Journal of Control, vol. 80, no. 7, pp. 1092-1108, 2007.

[40] L. Lapierre and D. Soetanto, "Nonlinear path-following control of an AUV," Ocean Engineering, vol. 34, no. 11, pp. 1734-1744, 2007.

[41] T. I. Fossen, Marine Control Systems: Guidance, Navigation, and Control of Ships, Rigs and Underwater Vehicles. Marine Cybernetics, 2002.

[42] J. Vervoort, Modeling and Control of an Unmanned Underwater Vehicle. $\mathrm{PhD}$ thesis, University of Canterbury, 2009.

[43] F. Borrelli, P. Falcone, T. Keviczky, J. Asgari, and D. Hrovat, "MPC based approach to active steering for autonomous vehicle systems," Int. J. Vehicle Autonomous Systems, vol. 3, no. 4, pp. 265-291, 2005.

[44] P. Falcone, F. Borrelli, J. Asgari, H. E. Tseng, and D. Hrovat, "Predictive active steering control for autonomous vehicle systems," IEEE Trans. on Control System Technology, vol. 15, no. 3, pp. 1-14, 2007.

[45] M. Bujarbaruah, X. Zhang, H. E. Tseng, and F. Borrelli, "Adaptive MPC for autonomous lane keeping," arXiv preprint, p. arXiv:1806.04335, 2018.

[46] J. B. Rawlings, D. Mayne, and M. M. Diehl, Model Predictive Control: Theory, Computation, and Design. Nob Hill Pub, 2009.

[47] V. Adetola, D. DeHaan, and M. Guay, "Adaptive model predictive control for constrained nonlinear systems," Syst. \& Contr. Letters, vol. 58, no. 5, pp. 320-326, 2009.

[48] I. Ullah, Y. Liu, X. Siu, and P. Kim, "Efficient and accurate target localization in underwater environment," IEEE Access, vol. 7, pp. 101415101426, 2019.

[49] I. Ullah, J. Chen, X. Su, C. Esposito, and C. Choi, "Localization and detection of targets in underwater wireless sensor using distance and angle based algorithms," IEEE Access, vol. 7, pp. 45693-45704, 2019. 
[50] J. Choi, J. Park, Y. Lee, J. Jung, , and H.-T. Choi, "Robust directional angle estimation of underwater acoustic sources using a marine vehicle," Sensors, vol. 18, no. 9, p. 3062, 2018.

[51] J. Choi and H.-T. Choi, "Multi-target localization of underwater acoustic sources based on probabilistic estimation of direction angle," in OCEANS, (Genova), pp. 1-6, 2015.

[52] H. Huang and Y. R. Zheng, "AoA assisted localization for underwater ad-hoc sensor networks," in OCEANS, (Monterey), pp. 1-6, 2016.

[53] M. Brandstein, J. Adcock, and H. Silverman, "A closed-form method for finding source locations from microphone-array time-delay estimates," in Conf. on Acoustics, Speech, and Signal Processing, pp. 3019-3022, 1995.

[54] T. Ballal and C. J. Bleakley, "3D location and orientation estimation using angle of arrival," in IEEE International Symposium on Intelligent Signal Processing, (Budapest, Hungary), pp. 21-26, 2009.

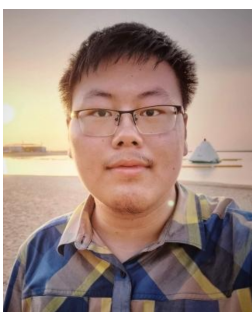

Ding Zhang received his B. Eng. degree in mechanical engineering from Huazhong University of Science and Technology, Wuhan, China, in 2018. He was a visiting student at the King Abdullah University of Science and technology, Jeddah, KSA from May to August in 2019. Now, he is pursuing his Ph.D. degree in Electronics and Computer Engineering at the Hong Kong University of Science and Technology, Kowloon, Hong Kong. He is interested in modeling, control and implementation of underwater wireless communication systems.

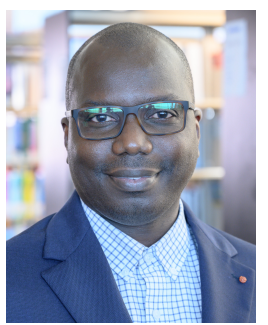

Ibrahima N'Doye is research scientist at King Abdullah University of Science and Technology (KAUST) in the Division of Computer, Electrical and Mathematical Sciences and Engineering (CEMSE). He received simultaneously the Ph.D. degree in Automatic Control from University of Lorraine at the Research Center of Automatic Control (CRAN-CNRS, University of Lorraine), France and University Hassan II Ain Chock, Casablanca, Morocco, in 2011. He was a Postdoc at the University of Luxembourg in the Faculty of Science, and with King Abdullah University of Science and Technology. His research interests are in the area of estimation and control systems engineering.

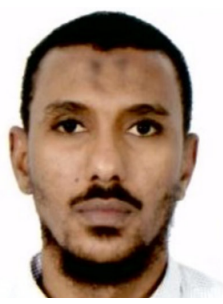

Tarig Ballal received the B.Sc. degree (Hons.) in electrical engineering from the University of Khartoum, Sudan, in 2001, the M.Sc. degree in telecommunications from the Blekinge Institute of Technology, Sweden, in 2005, and the Ph.D. degree from the School of Computer Science andInformatics, University College Dublin, Dublin, Ireland, in 2011. From 2011 to 2012, he was a Research Engineer with Resmed Sensor Technologies Ltd., and University College Dublin. During this period, he developed methods for non-contact breathing monitoring and classification from wireless biosensors data. Since 2012, he has been with the Electrical Engineering Department, King Abdullah University of Science and Technology, Saudi Arabia, where he is currently a Research Scientist. His current research focuses on regularization and robust estimation methods, image and signal processing, acoustic and electromagnetic sensing, and tracking and localization.His research interest also includes GNSS precise positioning and attitude determination, in addition to signal processing applications in wireless communications.

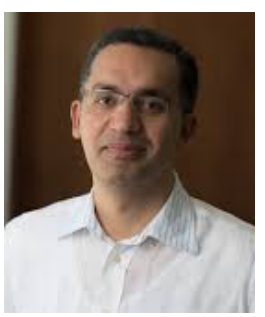

Tareq Al-Naffouri received the B.S. degrees in mathematics and electrical engineering (with first honors) from King Fahd University of Petroleum and Minerals, Dhahran, Saudi Arabia, the M.S. degree in electrical engineering from the Georgia Institute of Technology, Atlanta, in 1998, and the Ph.D. degree in electrical engineering from Stanford University, Stanford, CA, in 2004. He was a visiting scholar at California Institute of Technology, Pasadena, CA in 2005 and summer 2006. He was a Fulbright scholar at the University of Southern California in 2008 . He has held internship positions at NEC Research Labs, Tokyo, Japan, in 1998, Adaptive Systems Lab, University of California at Los Angeles in 1999, National Semiconductor, Santa Clara, CA, in 2001 and 2002, and Beceem Communications Santa Clara, CA, in 2004. He is currently an Associate Professor at the Electrical Engineering Department, King Abdullah University of Science and Technology (KAUST). His research interests lie in the areas of sparse, adaptive, and statistical signal processing and their applications, localization, machine learning, and network information theory. $\mathrm{He}$ has over 240 publications in journal and conference proceedings, 9 standard contributions, 14 issued patents, and 8 pending. Dr. Al-Naffouri is the recipient of the IEEE Education Society Chapter Achievement Award in 2008 and Al-Marai Award for innovative research in communication in 2009. Dr. Al-Naffouri has also been serving as an Associate Editor of Transactions on Signal Processing since August 2013.

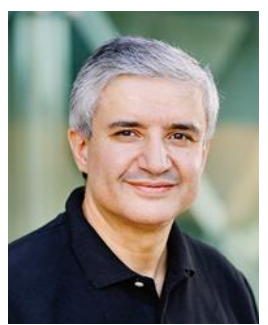

Mohamed-Slim Alouini (S'94-M'98-SM'03-F'09) was born in Tunis, Tunisia. He received the Ph.D. degree in Electrical Engineering from the California Institute of Technology (Caltech), Pasadena, CA, USA, in 1998. He served as a faculty member in the University of Minnesota, Minneapolis, MN, USA, then in the Texas A\&M University at Qatar, Education City, Doha, Qatar before joining King Abdullah University of Science and Technology (KAUST), Thuwal, Makkah Province, Saudi Arabia as a Professor of Electrical Engineering in 2009. His current research interests include the modeling, design, and performance analysis of wireless communication systems.

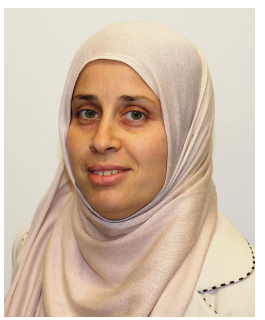

Taous Meriem Laleg-Kirati is an associate professor in the division of Computer, Electrical and Mathematical Sciences and Engineering at KAUST and member of the Computational Bioscience Research Center (CBRC). She joined KAUST in 2011 after being a postdoctoral fellow and a researcher at the French Institute for research in Computer Sciences and Control Systems (INRIA) in Bordeaux. She received her PhD in Applied Mathematics in 2008 from INRIA and Versailles University. Professor Laleg's work is in the general area of mathematical control theory, systems modeling, signal processing and their applications. Her primary research goals are directed towards developing effective estimation methods and algorithms to understand complex systems, extract hidden information and design control and monitoring strategies. Her research projects are motivated by real world problems in engineering and biomedical fields. She was elevated to an IEEE senior member in 2015. She won the best paper awards with her students at the IEEE International Conference of the IEEE Engineering in Medicine and Biology Society in 2018 and the IEEE International Conference on Control Engineering and Information Technology in 2015. She has been recognized for being among the three finalists in the academic of distinction category (engineering) of the leadership excellence for women awards in 2018. She is member of the IEEE Control Conferences Editorial Board (CEB) and a technical committee member at the International Federation of Automatic Control (IFAC). She is also member of the steering committee of KAUST women in data science. 